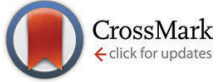

Cite this: Phys. Chem. Chem. Phys. $2016,18,26609$

Received 28th April 2016, Accepted 6th September 2016

DOI: $10.1039 / c 6 c p 02846 b$

www.rsc.org/pccp

\section{Influence of electric potential on the apparent viscosity of an ionic liquid: facts and artifacts $\dagger$}

\begin{abstract}
Moritz A. Ploss, ${ }^{a}$ Mark W. Rutland*bc ${ }^{\text {bnd Sergei Glavatskih }}{ }^{\text {ad }}$
According to recent findings, the steady shear viscosity of the ionic liquid 1-ethyl-3-methylimidazolium bis(trifluoromethylsulfonyl)imide ([Emim][Tf2N]) decreases significantly under the influence of electric potential. This implies a causal connection between nanoscale ordering at the electrified interface and a macroscopic change of transport properties. To study this phenomenon in more detail, we reproduced the above-mentioned measurements; however, we find no evidence that the viscosity of [Emim] [Tf2N] is a function of electric potential. Additionally, our results show that steady shear measurements can lead to artifacts that, at first glance, may appear to be potential-induced changes in viscosity. We demonstrate that the artifacts result from a sliding electrical contact at the working electrode of the electrochemical cell and we suggest to consider our findings for future viscosity measurements of ionic liquids.
\end{abstract}

\section{Introduction}

Room-temperature ionic liquids (IL) are molten salts consisting of two or more ionic species. Due to a large number of possible ion combinations, their physiochemical properties can be tuned widely. Consequently, they are often seen as designer solvents with an inherent potential to replace a variety of ecologically harmful liquids; still, high biodegradability and low ecotoxicity are yet to be demonstrated for most ILs. ${ }^{1-4}$

Research on ILs is currently conducted for various applications such as batteries, ${ }^{5-7}$ gas sensing and capturing, ${ }^{8-10}$ metal deposition $^{11}$ and lubrication technology. ${ }^{12}$ For these applications, IL transport properties, i.e., viscosity, diffusion coefficient and thermal/electrical conductivity, are important selection criteria. Thus, a major area of research is dedicated to designing and predicting these properties, ${ }^{13-16}$ and their partial interdependence has been demonstrated repeatedly. ${ }^{17-21}$ In consequence, controlling one of these properties may provide the means to influence others and constitute a route towards in situ control of IL-based systems.

In theory, in situ changes of IL properties can result from modifications of the ion orientation/distribution within the bulk and/or at a solid-liquid interface, ${ }^{22}$ usually due to external

\footnotetext{
${ }^{a}$ System and Component Design, KTH Royal Institute of Technology, SE-100 44 Stockholm, Sweden

${ }^{b}$ Surface and Corrosion Science, KTH Royal Institute of Technology, SE-100 44 Stockholm, Sweden. E-mail: mark@kth.se

${ }^{c}$ Chemistry, Materials and Surfaces, Box 5607, SE-114 86 Stockholm, Sweden

${ }^{d}$ Mechanical Construction and Production, Ghent University, B-900o Ghent, Belgium

$\dagger$ Electronic supplementary information (ESI) available: Experimental setup and procedure; additional experimental findings. See DOI: 10.1039/c6cp02846b
}

electric potential. The former is expected to influence the transport properties of the liquid significantly; yet experimental proof of potential-induced long-range ordering in the bulk structure has not been reported so far. ${ }^{23}$ In contrast to that, ordering in close proximity to the solid-liquid interface has been demonstrated in several empirical studies. Recently, Hjalmarsson et al. $^{24}$ have gained quantitative insights into the composition of the electrical double layer at a charged solid-liquid interface, and they show that the ratio of anions and cations can be altered by a change in electric potential. Before that, similar observations have been made by Hayes et $a .^{25,26}$ and Mezger et al., ${ }^{27,28}$ and a number of nanofriction experiments further support their findings. ${ }^{29-31}$

Accordingly, potential-induced structuring of ILs at solidliquid interfaces is well documented in the literature. However, a causal connection between the formation of nanoscale interfacial layers and a macroscopic change of transport properties has been reported only recently. Based on experiments with 1-ethyl-3-methylimidazolium bis(trifluoromethylsulfonyl)imide ([Emim][Tf2N]), Dold et al. ${ }^{32}$ claim that external electric potential can decrease the steady shear viscosity of [Emim][Tf2N] by as much as 15 to $20 \%$. They attribute their findings to a change in flow behavior close to a charged surface of their cone-on-plate rheometer, but they provide little detail on the process dynamics and the repeatability of their measurements.

To investigate these aspects in more detail, we reproduced the steady shear viscosity measurements by Dold et al. ${ }^{32}$ Additionally, we measured the complex viscosity of [Emim][Tf2N] in oscillation mode, which allows for a reduction in calibration overhead and measurement uncertainty. In the following, we present our findings together with a discussion of the measurement approach. 


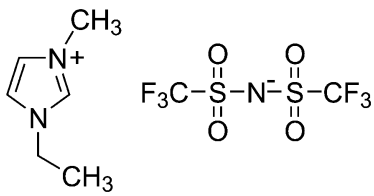

Fig. 1 Chemical structure of [Emim] [Tf2N].

\section{Methods}

\subsection{Materials}

IL of type [Emim][Tf2N] was bought from Ionic Liquids Technology $\mathrm{GmbH}$ (IoLiTec) and was used as delivered. According to the supplier, the IL's purity is greater $99.9 \%$ and the water content 64 ppm. Fig. 1 shows the chemical structure of the IL. Mineral oil of type Mineral Oil heavy by Sigma-Aldrich was used as a reference to identify systematic errors in the test setup and procedure.

\subsection{Experimental setup}

The experimental setup was devised based on the system of Dold et $a .^{32}+$ A rheometer of type DHR3 manufactured by TA Instruments was set up in a cone-on-plate viscometer configuration. During the experiments, the rotating stainless steel plate (diameter $20 \mathrm{~mm}$, cone angle $0^{\circ}$ ) was connected to a potentiostat and served as the working electrode (WE) of the electrochemical system. Counter (CE) and pseudo-reference (RE) electrode (both copper) were located at the bottom plate of the rheometer. Detailed information on the electrode design is provided in the ESI. $\dagger$

Measurements were performed both in rotation and oscillation mode. For the rotational measurements, Dold et al. ${ }^{32}$ provide no information on the electrical connection to the rotating steel plate (WE). Common technical solutions are sliding connectors as well as conductive liquids, although the latter may pose challenges in the context of IL.§ Thus, we contacted the WE using a sliding stranded copper wire. For comparison, a single-strand steel wire was used in later experiments. In oscillation mode, a single-strand copper wire was statically attached to the WE (no sliding). Other electrodes were contacted using soldering in both cases.

The electrical setup was driven by a CheapStat potentiostat. ${ }^{33}$ Control measurements were carried out using a homebuilt potentiostat. The potential difference between WE and CE was measured in situ using an Agilent 34420A Micro-Ohm Meter and all potentials are stated as measured. The notation "WE $\Rightarrow \mathrm{CE}$ " refers to the potential difference measured from WE to CE, i.e., the latter is connected to the ground potential. During the experiments, all electric potentials were set to be within the electrochemical window of the system. ${ }^{32}$ For polished electrodes, the open circuit potential (OCP) was measured to be in the order of $0.2 \mathrm{~V}$.

Prior to all measurements, electrodes were cleaned with acetone in an ultrasonic bath for 30 minutes, hand-cleaned with 2-propanol and dried in air.

¥ See Table ESI-1† for a detailed comparison with the setup of Dold et al. $\S$ The electrical resistance of the conductive liquid would need to be small in comparison to that of the IL.

\subsection{Experimental procedure, instrument readings}

For the rotational measurements, a sample of liquid was applied to the bottom plate of the rheometer and the top plate was lowered to a gap height of $0.5 \mathrm{~mm}$. Liquid was added/removed as required until the transition between the liquid's meniscus and the top plate was found to be smooth. After that, the following procedure was carried out at a shear rate of $300 \mathrm{~s}^{-1}$ (largest plate radius) and a bottom-plate temperature of $25{ }^{\circ} \mathrm{C}$ (room temperature $\approx 22^{\circ} \mathrm{C}$ ): (1) measure initial dynamic viscosity $\eta_{\text {in }}$ at OCP in rotation mode, (2) bring sliding wire in contact with rotating plate, (3) rotate plate until the wire-induced friction torque reaches a steady-state level, (4) measure dynamic viscosity at different electric potentials, (5) detach sliding wire from plate, (6) measure dynamic viscosity $\eta_{\text {fin }}$ at OCP.

A comparison of steps (1) and (6) can reveal permanent changes in viscosity that could be an indication of IL degradation due to electric potential. Also, a drift of OCP between steps (1) and (6) may indicate chemical reactions at one of the electrodes.

Step (3) is crucial to the accuracy of the measurements because the setup only allows to measure a relative change in viscosity. This is a consequence of the sliding electrical contact to the WE that induces a friction torque on the rotating plate. Since the viscosity is directly calculated from the torque $T$ that is required to rotate the plate, ${ }^{34} T$ needs to be resolved into a viscosity-induced factor $T_{\eta}$ and a friction-induced factor $T_{\mathrm{f}}$ in order to correct for the wire friction - especially in the case of low-viscosity liquids $\left(T_{\mathrm{f}} \gg T_{\eta}\right)$. Hence, the dynamic (shear) viscosity $\eta$ is calculated as

$$
\eta=\frac{2 h\left(T-T_{\mathrm{f}}\right)}{\pi \Omega r^{4}}=\frac{2 h\left(T_{\eta}\right)}{\pi \Omega r^{4}}=\eta^{\circ}-\eta_{\mathrm{f}}^{\circ},
$$

where $h$ is the gap height between the plates, $r$ is the plate radius and $\Omega$ is the angular velocity of the plate. The uncorrected measurement output of the instrument (the signal that contains both $T_{\eta}$ and $T_{\mathrm{f}}$ ) will be referred to as $\eta^{\circ}$ in the following; the component of $\eta$ that can be attributed to the wire friction will be referred to as the friction-torque-equivalent dynamic viscosity $\eta_{\mathrm{f}}^{\circ}$ and was in the order of 60 to $220 \mathrm{mPa}$ s if not stated otherwise.

The wire-induced friction torque varies with time (due to run-in), sliding speed and magnitude of normal (wire) force, and it needs to be monitored continuously. Because of that, all rotational measurements were carried out over a period of at least $90 \mathrm{~s}$ and it was found that a timespan of around $3 \mathrm{~min}$ should be given for the signal to return to a steady-state level between consecutive test runs.

In case of the oscillation measurements, the small spring force exerted by the electrical connection to the WE proved to have negligible impact on the measured torque. Therefore, no correction for wire-induced losses was required $\left(\eta^{\circ}=\eta, \eta_{\mathrm{f}}^{\circ}=0\right)$, which allows to omit step (3) of the above procedure and to present the results as measured. Since the steady shear viscosity cannot be measured in oscillation mode, the complex viscosity $\eta^{*}$ was measured instead. The oscillation parameters were chosen according to the Cox-Merz rule which is expected to hold true for Newtonian fluids. ${ }^{35}$ Accordingly, it is assumed that 
$\eta(\dot{\gamma})=\eta^{*}(\omega)$, where $\dot{\gamma}$ and $\omega$ are the (maximum) shear rate in rotational mode and the angular frequency in oscillation mode. The oscillation amplitude was ramped linearly to identify stable operation points.

\section{Results}

\subsection{Rotation measurements at open circuit potential}

Table 1 shows the results for the initial and final viscosity measurements (step (1) and (6), see above procedure) at OCP. All measurements were repeated at least three times. As can be seen, no persistent change in the dynamic viscosity is observed between step (1) and (6), hence the signal shows no indication for IL degradation or viscosity changes due to water uptake. The measurement error can be attributed to slight fluctuations in room temperature and meniscus geometry. Although our results generally agree with literature ${ }^{36,37}$ and supplier data, we cannot explain the wide discrepancy between our findings and those of Dold et al., ${ }^{32}$ who measured the dynamic viscosity of [Emim][Tf2N] to be in the order of $600 \mathrm{mPa} \mathrm{s}$ - a difference of factor 18 .

\subsection{Rotation measurements with applied potential}

As described above, a sample of [Emim][Tf2N] was applied to the rheometer and the viscosity reading $\eta^{\circ}$ was recorded under the influence of electric potential. Depending on the sliding wire material, the viscosity signal was allowed to stabilize during an initial run-in period of varying duration. $\uparrow$ Afterwards, it can be seen that the application of negative electric potential leads to a decrease in the viscosity reading $\eta^{\circ}$ (Fig. 2 and 3 ) that is quickly recovered once the external potential is no longer enforced (Fig. 3). The magnitude of change is found to be positively correlated with the magnitude of negative potential, and no change in the viscosity reading is observed for positive potentials in the case of a copper wire sliding connection. One measurement (see Fig. 3) shows a decrease in the viscosity reading by up to $38 \mathrm{mPa}$, which is greater than the viscosity of [Emim][Tf2N] at OCP. This means that if the change in the viscosity reading was caused by an actual change in liquid viscosity, the viscosity of [Emim][Tf2N] would need to decrease to a negative value once potential is applied.

In contrast to these observations, rotation measurements with dielectric mineral oil show no effect of electric potential on the viscosity reading in the range of $\pm 1650 \mathrm{mV}$.

Additional sample measurements of the relationship between electric potential and current indicate that the system behaves like an IL double-layer capacitor in the case of [Emim] $[\mathrm{Tf} 2 \mathrm{~N}]{ }^{38-40}$ Measurements of the electric current at the sliding wire connection show an initial peak current (in the order of $\pm 1 \mathrm{~mA}$ ) once potential of $\pm 1650 \mathrm{mV}$ is applied. After that, a slow current decay to a constant value (about $\pm 0.05 \mathrm{~mA}$ ) is observed, which we assume to be caused by interface relaxation processes and Faradaic currents as reported by Zeng et $a l^{40}$ for other ILs with imidazolium cations and bis(trifluoromethylsulfonyl)imide anions.

T See Section 4.2 for more information on the effect of run-in time and sliding wire material.
Table 1 Results of initial $\left(\eta_{\text {in }}\right)$ and final $\left(\eta_{\text {fin }}\right)$ viscosity measurements at $25^{\circ} \mathrm{C}$ and OCP compared to literature data $\left(\eta_{\text {lit }}\right)$. All data are rounded to the nearest integer number; the $\eta_{\text {lit }}$-value reported for [Emim][Tf2N] was measured for a water content of $30 \mathrm{ppm}$ by Makino et al. ${ }^{36}$

\begin{tabular}{lccl}
\hline Liquid & $\eta_{\text {in }}(\mathrm{mPa} \mathrm{s})$ & $\eta_{\text {fin }}(\mathrm{mPa} \mathrm{s})$ & $\eta_{\text {lit }}(\mathrm{mPa} \mathrm{s})$ \\
\hline [Emim][Tf2N] & $33 \pm 1$ & $33 \pm 1$ & 33 \\
Mineral oil heavy & $142 \pm 1$ & $142 \pm 1$ & $\mathrm{n} / \mathrm{a}$
\end{tabular}

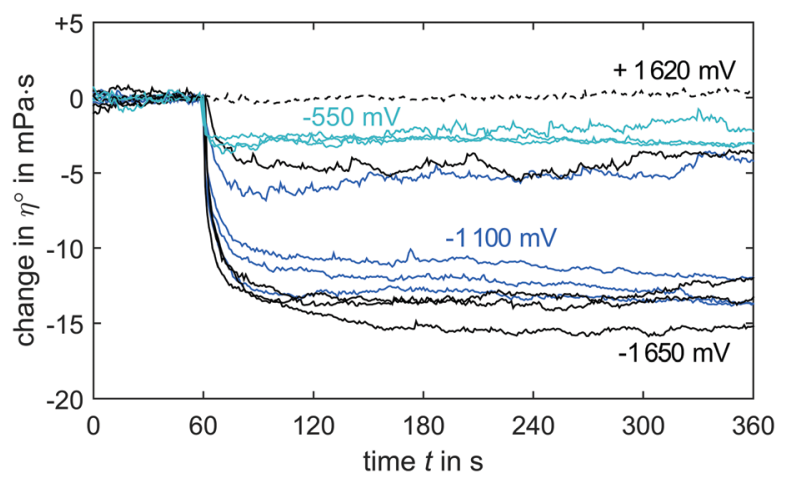

Fig. 2 Change in viscosity reading $\eta^{\circ}$ for different electric potentials. All potentials were applied from $t=60 \mathrm{~s}$ to $t=360 \mathrm{~s}$ and the results were obtained during one consecutive test run. $\eta_{\text {ocp }}^{\circ}$ is between 106 and $118 \mathrm{mPa} s$ for all experiments.

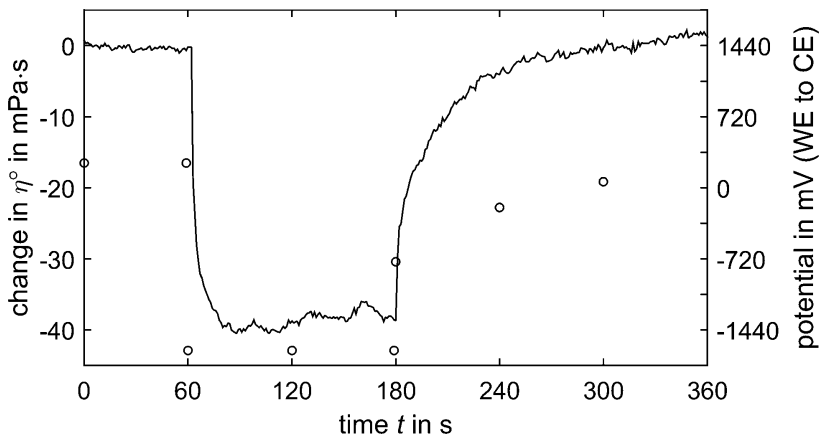

Fig. 3 Viscosity reading $(-)$ and potential $(O)$ over test time. Potential of $-1650 \mathrm{mV}$ was applied from $t=60 \mathrm{~s}$ to $t=180 \mathrm{~s}$. The average drop in the viscosity reading is $38 \mathrm{mPa}$, the viscosity of [Emim] [Tf2N] at OCP is $33 \pm 1 \mathrm{mPa} s, \eta_{\text {ocp }}^{\circ}=216 \mathrm{mPa} \mathrm{s}$.

Moreover, small-scale material deposition between electrodes (anode to cathode, for both copper/steel depending on polarity) could further contribute to the net current. Consequently, significant electric current flow occurs at the sliding wire connection over the entire duration of the measurements. Once the potential is no longer enforced, the open electric circuit leads to a slow decay (leakage) of the potential difference between WE and CE as shown in Fig. 3.\|

\subsection{Oscillation measurements with applied potential}

To investigate if potential-induced changes in the viscosity reading can be observed in oscillation mode, the complex

\| Therefore, current flow at the sliding wire occurs only while external potential is applied, and the leakage current does not flow through the wire since the circuit is not closed during discharge. 


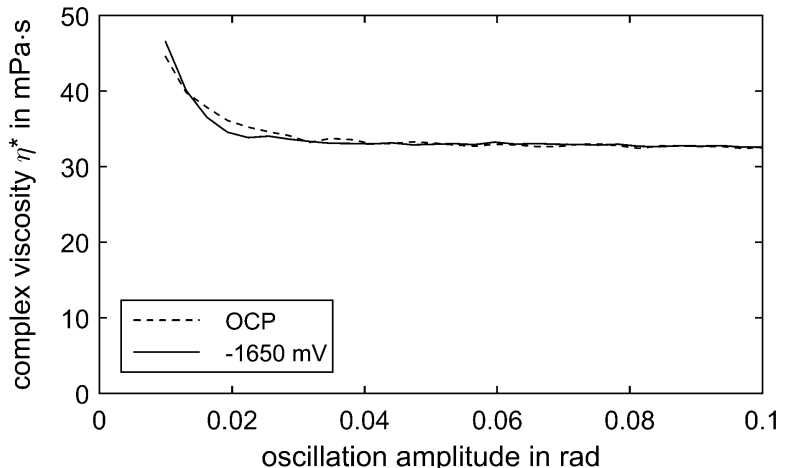

Fig. 4 Viscosity measurements of [Emim][Tf2N] in oscillation mode at OCP and $-1650 \mathrm{mV}$. Potential was applied over the entire duration of the measurement. For oscillation amplitudes $>0.03$ rad the complex viscosity is constant within $33+1.5 \mathrm{mPa} s$ independent of the external electric potential.

viscosity $\eta^{*}$ of $[$ Emim] [Tf2N] was measured at OCP and $-1650 \mathrm{mV}$. As can be seen in Fig. 4, identical values $\left(\eta^{*} \approx\right.$ $33 \mathrm{mPa} \mathrm{s}$ ) are obtained in both cases and no influence of electric potential is found.

\section{Discussion}

\subsection{Change in friction torque vs. change in viscosity}

The above experiments demonstrate that applying electric potential in rotational viscosity measurements of [Emim][Tf2N] can cause a systematic decrease in the instrument's viscosity reading $\eta^{\circ}$. Repeatability is poor when comparing different test runs (Fig. 2 and 3) and the viscosity reading is observed to drop by more than the baseline viscosity of [Emim][Tf2N]. Moreover, no evidence is found that the viscosity of $[\mathrm{Emim}][\mathrm{Tf} 2 \mathrm{~N}]$ is a function of electric potential in oscillation mode. Thus, we conclude that the observed changes in the viscosity reading are caused by a measurement artifact rather than a change in IL properties.

We propose that the structure of the material transfer film between sliding wire and rotating plate is altered by electric current flow, which leads to a reduction in sliding friction at the contact point. The underlying electro-tribological mechanism is described in much detail in Section 4.2 based on the work of Braunovic, Myshkin and Belyi. ${ }^{41-43}$ It follows that the decrease in the viscosity reading can be attributed to a decrease in the friction-induced torque $T_{\mathrm{f}}$ rather than the viscosity-induced torque $T_{\eta}$ (see eqn (1)).

To demonstrate the effect of electric current on the friction torque at the sliding-wire connection, the experimental setup was adapted as shown in Fig. ESI- $2 . \dagger$ An air gap of $0.5 \mathrm{~mm}$ was set between top and bottom plate, the top plate was left to rotate in ambient air at a maximum shear rate of $300 \mathrm{~s}^{-1}$ and was contacted with a sliding wire (steel). To close the electric circuit, a second sliding wire (steel) was brought into contact with the rotating plate and the induced friction torque was adjusted to be of similar magnitude compared to the above experiments $(2 \times 170 \mathrm{mPa} \mathrm{s})$. When potential is applied to this

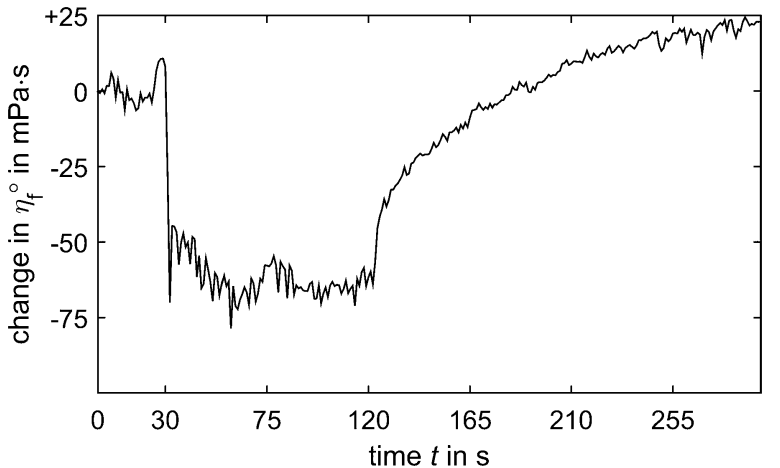

Fig. 5 Change in friction-torque-equivalent viscosity $\eta_{\mathrm{f}}^{\circ}$ due to electric potential from $t=30 \mathrm{~s}$ to $t=123 \mathrm{~s}, \eta_{\mathrm{f}, \text { ocp }}^{\circ} \approx 320 \mathrm{mPa}$. The reduction in friction torque is caused by an immediate decrease of the sliding friction coefficient at the electrical connection to the WE. The presence of a second sliding wire and absence of IL leads to an overall increase in signal noise.

system, the viscosity reading is observed to decrease by 15 to $20 \%$ as can be seen in Fig. 5 . Here, both the magnitude of change as well as the curve progression agree well with the findings in Fig. 2 and 3.**

In the measurements with [Emim][Tf2N], the electric current originates from the instantaneous potential step at the IL capacitor. Hence, it is expected to be linked to the capacitance of the system. In consequence, although the above findings should be seen as qualitative rather than quantitative, the underlying mechanism can be expected to apply likewise for more sophisticated setups and to be (qualitatively) similar for a range of electrode materials. Because of that, we suggest to measure the electro-rheological properties of IL in oscillation mode, which allows to avoid the above artifacts and to significantly reduce the calibration overhead.

\subsection{Influence wire material}

Although our results for stranded copper wires and singlestrand steel wires are in general agreement, some differences between these two setups were observed. When the sliding electrical contact to the WE was made using a stranded copper wire, a distinct pattern was found during all experiments. Initially, after a sample of [Emim][Tf2N] was applied to the rheometer and the viscosity reading $\eta^{\circ}$ was recorded under the influence of electric potential, no changes in the viscosity reading were observed for both positive and negative potentials as shown in Fig. ESI-3. $\dagger$ However, as the rheometer was left to shear the sample continuously at a shear rate of $300 \mathrm{~s}^{-1}$ for several hours (sliding wire attached, no potential applied), artifacts started to appear as a reaction to external electric potential. It was found that changes in the viscosity reading $\eta^{\circ}$ (i.e., measurement artifacts) were only observed for long run-in times $(6 h+)$ and the magnitude of the artifact increased with run-in time.

** Note that, for convenience, the potential-induced changes in the friction torque are reported in units of viscosity although no liquid is present in the case of Fig. 5. 
Table 2 A schematic representation of the findings of Belyi, Konchits and Savkin $^{41-43}$ for copper-containing composite-metal brushes

\begin{tabular}{ll}
\hline Run-in state & Resistance brush $(+/-)$ \\
\hline No run-in & High/high \\
Film system formed & Medium/medium high \\
Film removed by neg. brush & $-/$ low
\end{tabular}

In experiments with sliding copper brushes, Belyi, Konchits and Savkin ${ }^{43}$ find that the current state of run-in has significant influence on the electrical contact resistance of metal-containing brushes. In the case of (copper and silver) metal-polymer brushes sliding against a copper collector ring, they link their findings to the formation of a $\mathrm{Cu}_{2} \mathrm{O}$ transfer film system between the contacting bodies. Without this system, the contact resistance is found to be comparably high independent of the brush polarity. Once the system was formed, however, the electrical resistance reduces for both brush polarities. In their experiments, a larger decrease can be observed for cathodic (-) brushes compared to anodic (+) brushes. A simplified summary of this process is presented in Table 2.

An explanation for these observations is found in the semiconductive nature of the transfer film system, which changes as a function of run-in time: (1) In the beginning, when an intact $\mathrm{Cu}_{2} \mathrm{O}$-layer is found beneath the transfer film, current is mostly transferred by first passing the transfer layer and then by "fritting" ${ }^{43}$ the underlying $\mathrm{Cu}_{2} \mathrm{O}$-layer. (2) During later stages, when the $\mathrm{Cu}_{2} \mathrm{O}$-layer is damaged or partially removed due to wear, the current is transferred through a double layer of transfer film material and exposed patches of $\mathrm{Cu}$ base material. Comparing these two pathways, it becomes evident that in the first case $\left(\mathrm{Cu}_{2} \mathrm{O}\right.$-transfer-film-layer $)$, the overall resistance is comparably high as the fritting process constitutes an additional resistance that needs to be overcome. Compared to that, less $\mathrm{Cu}_{2} \mathrm{O}$ is present in the second case and the resistance of the contact depends mostly on the resistance of the transfer film itself. It follows that the lowest contact resistance can be achieved by (1) initial removal of high resistance $\mathrm{Cu}_{2} \mathrm{O}$ boundary layers during run-in and (2) subsequent exposure of $\mathrm{Cu}$-patches by removal of the transfer films. ${ }^{43}$

Belyi et al. show that step (2) can be achieved by the application of external electric potential, since a cathodic (-) brush "tends to clean the counterface of foreign particles". ${ }^{43}$ Consequently, the magnitude of current at a given contact increases immediately if the potential is switched from its open circuit value to a negative potential, given that the initial $\mathrm{Cu}_{2} \mathrm{O}$ layer was damaged during run-in.

As a result, a low resistance pathway through the sliding contact is established. However, the reduction in contact resistance does not yet explain the reduction in the sliding friction coefficient observed in our experiments. To explain this "lubricating action of electric current", ${ }^{41}$ Braunovic, Konchits and Savkin argue that the passage of current at the sliding contact leads to an increase in contact temperature together with material weakening and subsequent surface modification. In their experiments, they find a significant and immediate decrease in friction coefficient for a cathodic (-) brush under passage of current, ${ }^{42}$ and the results closely resemble those shown in Fig. 2. In addition to that, they underline that the observations are independent of the brush-collector material configuration, i.e., similar effects can be observed if collector and brush material are switched. ${ }^{41}$

Thus, in our case of a stranded copper wire sliding against a rotating steel plate, we assume that long run-in times are required in order to remove the oxide layer on the $\mathrm{Cu}$-wire by slow wear under low contact pressure. By that, patches of non-oxidized $\mathrm{Cu}$ are exposed and immediately covered by a protective transfer layer. When applying an external potential, the cathodic (-) brush helps to remove parts of the transfer film, which in turn changes the friction coefficient and lowers the contact resistance to that of the exposed $\mathrm{Cu}$-surface. As a result, significant current passes the contact, which may result in additional heat generation. This may lead to small-scale material decay and related changes in the tribological behavior.

Due to a lack of transfer film and exposed $\mathrm{Cu}$, we assume that the above process does not occur after short run-in times. Thus, although the resistance of the sliding contact is low enough for current to pass in early stages of the experiment, the tribological interface does not change significantly during current passage since no transfer film was formed yet.

Finally, once the circuit is opened and the external potential is no longer enforced, the current at the sliding contact no longer flows. As a result, the friction coefficient slowly recovers to its initial value as the transfer film reforms.

In the case of the sliding steel wire, a different trend was observed in our experiments: at the beginning of the experiment (no run-in required), the artifacts were well reproducible, but soon seemed to fade with increasing run-in time and contact oxidation. Also, in contrast to observations with the copper wire, the artifacts were well reproducible for both positive and negative potentials, but again solely dependent on the magnitude of the final external potential.

In the case of the steel wire, advancing contact oxidation can be expected to increase the electrical resistance of the contact with time.$^{44}$ Experimental observations confirm that black wear debris accumulates on the sliding track if the steel wire slides against the plate, while the copper wire only leaves polishing marks and a thin transfer film due to the low contact pressure. In addition to that, for the setup shown in Fig. ESI-2, $\dagger$ application of potential leads to no current flow at the steel sliding contact for run-in times of more than one hour.

In consequence, current-induced artifacts can only be observed during the run-in phase for the steel-steel sliding contact. The process evolution can be described as follows: during the initial severe wear process, protective boundary layers are removed, which allows for the formation of highly conductive metalto-metal contacts. Because stable transfer films cannot form during run-in, they have minor impact on the contact resistance of the sliding contact. Hence, the magnitude of electric current flow is similar for positive and negative potentials, which causes the friction coefficient to change by the same magnitude for both polarities. Again, the decrease in sliding friction may be 
attributed to both the removal of particles as well as heat generation. As the contact resistance increases with advancing contact oxidation, the electric current (for a given potential) vanishes and artifacts can no longer be observed.

\section{Conclusions}

Based on our analysis, we reach the following conclusions:

- No evidence is found that the viscosity of [Emim][Tf2N] as measured in a cone-on-plate rheometer - is a function of electric potential within the given potential range.

- All observed potential-induced changes in the instrument's viscosity reading can be fully attributed to a measurement artifact. The artifact results from an electric current that passes the sliding-wire connection to the working electrode. The current is caused by an instantaneous potential step at the IL doublelayer capacitor and occurs well within the electrochemical window of the cell.

- No artifacts are observed in oscillation mode. Thus, we suggest to measure the viscosity of ILs in oscillation mode if a sliding connection to the rotating plate cannot be avoided.

\section{Acknowledgements}

The authors acknowledge financial support by the Knut and Alice Wallenberg Foundation as part of project KAW2012.0078 and the Swedish Research Council as part of project 2014-4694. Special thanks go to Patrick Rohlmann for the preliminary modification of the rheometer system, to Bulat Munavirov and Govind Kumar Prajapati for helpful discussions, and to Ju Shu for a short survey of the Chinese literature on electro-tribology.

\section{References}

1 M. Deetlefs and K. R. Seddon, Green Chem., 2010, 12, 17-30.

2 M. Petkovic, K. R. Seddon, L. P. N. Rebelo and C. Silva Pereira, Chem. Soc. Rev., 2011, 40, 1383-1403.

3 R. N. Das and K. Roy, Mol. Diversity, 2013, 17, 151-196.

4 M. C. Bubalo, K. Radošević, I. R. Radojčić, J. Halambek and V. G. Srček, Ecotoxicol. Environ. Saf., 2014, 99, 1-12.

5 E. Quartarone and P. Mustarelli, Chem. Soc. Rev., 2011, 40, 2525-2540.

6 M. D. Bhatt, H. Geaney, M. Nolan and C. O'Dwyer, Phys. Chem. Chem. Phys., 2014, 16, 12093-12130.

7 M. D. Bhatt and C. O'Dwyer, Phys. Chem. Chem. Phys., 2015, 17, 4799-4844.

8 D. S. Silvester, Analyst, 2011, 136, 4871-4882.

9 X. Zhang, X. Zhang, H. Dong, Z. Zhao, S. Zhang and

Y. Huang, Energy Environ. Sci., 2012, 5, 6668-6681.

10 A. Rehman and X. Zeng, RSC Adv., 2015, 5, 58371-58392.

11 A. P. Abbott and K. J. McKenzie, Phys. Chem. Chem. Phys., 2006, 8, 4265-4279.

12 F. Zhou, Y. Liang and W. Liu, Chem. Soc. Rev., 2009, 38, 2590-2599.
13 R. L. Gardas and J. A. Coutinho, Fluid Phase Equilib., 2008, 266, 195-201.

14 E. I. Izgorodina, Phys. Chem. Chem. Phys., 2011, 13, 4189-4207.

15 J. A. P. Coutinho, P. J. Carvalho and N. M. C. Oliveira, $R S C$ Adv., 2012, 2, 7322-7346.

16 S. T. Keaveney, J. B. Harper and A. K. Croft, RSC Adv., 2015, 5, 35709-35729.

17 W. Xu, E. I. Cooper and C. A. Angell, J. Phys. Chem. B, 2003, 107, 6170-6178.

18 H. A. Every, A. G. Bishop, D. R. MacFarlane, G. Oradd and M. Forsyth, Phys. Chem. Chem. Phys., 2004, 6, 1758-1765.

19 K. J. Fraser, E. I. Izgorodina, M. Forsyth, J. L. Scott and D. R. MacFarlane, Chem. Commun., 2007, 3817-3819.

20 K. R. Harris, T. Makino and M. Kanakubo, Phys. Chem. Chem. Phys., 2014, 16, 9161-9170.

21 T. Yamaguchi, T. Yonezawa and S. Koda, Phys. Chem. Chem. Phys., 2015, 17, 19126-19133.

22 R. Capozza, A. Benassi, A. Vanossi and E. Tosatti, J. Chem. Phys., 2015, 143, 144703.

23 R. Hayes, G. G. Warr and R. Atkin, Chem. Rev., 2015, 115, 6357-6426.

24 N. Hjalmarsson, D. Wallinder, S. Glavatskih, R. Atkin, T. Aastrup and M. W. Rutland, Nanoscale, 2015, 7, 16039-16045.

25 R. Hayes, N. Borisenko, M. K. Tam, P. C. Howlett, F. Endres and R. Atkin, J. Phys. Chem. C, 2011, 115, 6855-6863.

26 R. Hayes, N. Borisenko, M. K. Tam, P. C. Howlett, F. Endres and R. Atkin, J. Phys. Chem. C, 2011, 115, 6855-6863.

27 M. Mezger, H. Schröder, H. Reichert, S. Schramm, J. S. Okasinski, S. Schöder, V. Honkimäki, M. Deutsch, B. M. Ocko, J. Ralston, M. Rohwerder, M. Stratmann and H. Dosch, Science, 2008, 322, 424-428.

28 M. Mezger, S. Schramm, H. Schröder, H. Reichert, M. Deutsch, E. J. De Souza, J. S. Okasinski, B. M. Ocko, V. Honkimäki and H. Dosch, J. Chem. Phys., 2009, 131, 094701.

29 J. Sweeney, F. Hausen, R. Hayes, G. B. Webber, F. Endres, M. W. Rutland, R. Bennewitz and R. Atkin, Phys. Rev. Lett., 2012, 109, 155502.

30 H. Li, M. W. Rutland and R. Atkin, Phys. Chem. Chem. Phys., 2013, 15, 14616-14623.

31 H. Li, P. K. Cooper, A. E. Somers, M. W. Rutland, P. C. Howlett, M. Forsyth and R. Atkin, J. Phys. Chem. Lett., 2014, 5, 4095-4099.

32 C. Dold, T. Amann and A. Kailer, Phys. Chem. Chem. Phys., 2015, 17, 10339-10342.

33 A. A. Rowe, A. A. H. Padua, J. Bonham, R. J. White, M. P. Zimmer, R. J. Yadgar and T. Hobza, et al., PLoS One, 2011, 6, e23783.

34 S. Raha, M. J. Williams and P. Lamb, J. Phys. E: Sci. Instrum., 1968, 1, 1113-1115.

35 S.-P. Li, G. Zhao and H.-Y. Chen, J. Dispersion Sci. Technol., 2005, 26, 415-419.

36 T. Makino, M. Kanakubo, Y. Masuda, T. Umecky and A. Suzuki, Fluid Phase Equilib., 2014, 362, 300-306.

37 J. Jacquemin, P. Husson, A. A. H. Padua and V. Majer, Green Chem., 2006, 8, 172-180. 
38 T. Sato, G. Masuda and K. Takagi, Electrochim. Acta, 2004, 49, 3603-3611.

39 M. Ue, M. Takeda, A. Toriumi, A. Kominato, R. Hagiwara and Y. Ito, J. Electrochem. Soc., 2003, 150, A499-A502.

$40 \mathrm{X}$. Zeng, in Electrochemistry in Ionic Liquids - Volume 1: Fundamentals, ed. A. A. J. Torriero, Springer International Publishing, 2015, ch. 2, p. $24 \mathrm{ff}$.
41 M. Braunovic, V. V. Konchits and N. K. Myshkin, Electrical Contacts - Fundamentals, Applications and Technology, CRC Press, 2006, p. 473ff.

42 N. Myshkin and V. Konchits, Wear, 1992, 158, 119-140.

43 V. Belyi, V. Konchits and V. Savkin, Wear, 1982, 78, 249-258.

44 H. Kato, Tribol. Int., 2008, 41, 735-742. 